\title{
Nivel de satisfacción en pacientes del servicio de oftalmología de un hospital de nivel II en los años 2014 y 2018, y su relación con calidad global
}

\section{Satisfaction levels in patients attending an ophthalmology service in a level II hospital between 2014 and 2018, and their relationship with overall quality}

Correspondencia Nora Beatriz Mendoza Díaz noritabeatriz@gmail.com

\section{Recibido: 21/11/2019}

Arbitrado por pares

Aprobado: 05/02/2020

\section{Citar como: Mendoza NB,}

Placencia M. Nivel de satisfacción en pacientes del servicio de oftalmología de un hospital de nivel II en los años 2014 y 2018, y su relación con calidad global. Acta Med Peru. 2020;37(1):19-26. doi: https://doi.org/10.35663/ amp.2020.371.964
Nora B. Mendoza1, Maritza Placencia²

Hospital II Vitarte, Seguro Social de Salud del Perú. Lima, Perú.

Facultad de Medicina, Universidad Nacional Mayor de San Marcos. Lima, Perú

\section{RESUMEN}

Objetivo: comparar el nivel de satisfacción en los pacientes con la calidad de atención brindada en el servicio de oftalmología de un hospital de segundo nivel de atención entre los años 2014 y 2018, además determinar la calidad global en el 2018. Materiales y métodos: se realizó el estudio observacional, transversal, analítico. Se consideró una muestra de 678 pacientes y se utilizaron dos instrumentos: SERVQUAL que precisa el nivel de satisfacción en cinco dimensiones de calidad y SERVQHOS que mide la satisfacción por la calidad global del servicio recibido. Resultados: en el año 2014 la población ( $n=346)$ encuestada con SERVQUAL evidenció el nivel de "satisfecho" con la calidad de atención recibida, destacando la dimensión de seguridad; pero en el año 2018, la población estudiada (n=332) mostró que los niveles de satisfacción disminuyeron en todas sus dimensiones. Respecto al SERVQHOS los pacientes estuvieron satisfechos en su mayoría, con la calidad global y recomendarían este hospital a otras personas, su ingreso fue con el tiempo necesario, los profesionales y personal sanitario les hicieron pruebas con su permiso, conocen el nombre de su médico y reciben suficiente información sobre su estado de salud. Se determinó una débil asociación entre SERVQUAL y SERVQHOS directamente proporcional entre el nivel de satisfacción de los pacientes y la calidad global. Conclusión: en el 2014 los pacientes evidenciaron el nivel de "satisfecho" con la calidad de atención recibida comparado al 2018 que fue "levemente insatisfecho", y en la medición de la calidad global estuvieron satisfechos destacándose la atención del médico y el respeto mostrado a los pacientes.

Palabras clave: Satisfacción del paciente; Calidad de la atención; Servicios oftalmológicos (fuente: DeCS-BIREME). 


\begin{abstract}
Objective: to compare satisfaction levels of patients regarding quality of care in the ophthalmology service in a level II hospital between 2014 and 2018, and also, to determine overall quality in 2018. Materials and methods: this is an observational, cross section and analytical study. The sample comprised 678 patients, and two instruments were used: SERVQUAL, which measures satisfaction levels in five quality dimensions, and SERVQHOS, which measures the overall quality of services used. Results: in 2014, subjects ( $n=346$ ) interviewed with the SERVQUAL instrument declared being "satisfied" with the quality of care they received, particularly with respect to safety; but, in 2018, subjects $(n=332)$ declared their satisfaction levels were reduced in all scenarios. With respect to SERVQHOS, most subjects declared being satisfied with the overall quality of services and said they would recommend this hospital to other persons. Their encounters had enough time, healthcare personnel performed tests after asking for permission, subjects knew the names of physicians taking care of them, and they also said they received adequate information about their health condition. A weak association between SERVQUAL and SERVQHOS was found, which was directly proportional between patient satisfaction levels and overall quality. Conclusion: during 2014, patients declared "being satisfied" with the quality of care they received compared to 2018, when they declared being "mildly unsatisfied". When overall quality was measured, patients declared being satisfied, emphasizing medical care and respect towards them.
\end{abstract}

Keywords: Patient satisfaction; Quality of health care; Ophthalmologic services (source: MeSH NLM).

\section{INTRODUCCIÓN}

La Organización Panamericana de la Salud (OPS) define a un servicio de prestación de salud como aquel conjunto de características de un servicio que permiten satisfacer las necesidades y expectativas del usuario; si este proceso es de calidad, existe más chance que de los usuarios sigan las indicaciones médicas. En el Perú, el Sistema de Gestión de la calidad en Salud ${ }^{[1]}$, dependencia del Ministerio de Salud (MINSA), tiene la finalidad de mejorar continuamente los servicios, recursos y tecnologías sanitarias mediante el desarrollo de una cultura de calidad que considere las necesidades y expectativas de los usuarios, usando como estrategia la investigación operativa, la investigación gerencial y el uso de herramientas de medición de la calidad como el SERVQUAL ${ }^{[2]}$ y su versión modificada en español ${ }^{[3]}$.

El programa de gestión de la calidad del Seguro Social del Perú (EsSalud) ${ }^{[4]}$ declara que el compromiso de la institución es dar el mejor servicio "con los atributos de disposición, profesionalismo y calidez que puedan llevarse a la práctica, plasmándolos en rutinas y conductas observables que los hagan visibles y tangibles para los asegurados, en los diferentes servicios", declaración que integra el concepto de calidad en la prestación de servicios médicos medida por el usuario externo. Donabedian Avedis ${ }^{[5]}$ fue quién fundamentó la relación directa de la calidad de atención sanitaria ofrecida con la estructura (elementos tangibles institucionales) y los procesos (actividades administrativas y sanitarias en el acto médico); y del resultado de esta gestión sanitaria con la satisfacción del usuario.

Parasuraman, Zeithaml y Berry ${ }^{[2,6]}$ crearon el instrumento SERVQUAL que permite medir la calidad de servicio percibida a través del cálculo de las diferencias entre percepciones y expectativas, las cuales se traducen en indicadores de oportunidad de mejora continua que se incorporarán en la gestión de estos procesos ${ }^{[7-12]}$. Otro instrumento similar es el SERVQHOS ${ }^{[13]}$, el cual difiere del anterior en la organización de las preguntas, agrupándolas en variables relacionadas con la calidad objetiva (infraestructura, bienestar, dotación de equipos) y lo relacionado con la calidad subjetiva (lo que el usuario percibe a propósito del trato, comunicación e información por parte del personal de salud, quién posibilita establecer una relación empática con los pacientes) y daría una valoración real de la calidad de todo el proceso de atención ${ }^{[13,14]}$

El Hospital II Vitarte de EsSalud tiene una población adscrita de asegurados de 168273 pacientes y atiende referidos de los centros asistenciales de Casapalca, San Mateo, Matucana, Chosica, Voto Bernales, y Huaycán. Durante el periodo 2014 2018 hubo cambios en la gestión administrativa institucional que requieren de información sobre la calidad de los servicios ofertados; en el servicio de Oftalmología aún no tenemos una investigación disponible que nos permita tomar acciones de mejora, por lo que se planteó determinar el nivel de satisfacción de los pacientes atendidos en oftalmología en el año 2014 comparado con el 2018; y la medición de satisfacción con calidad global en el año 2018, sus resultados permitirían establecer las recomendaciones y oportunidades de mejora continua.

\section{MATERIAL Y MÉTODOS}

\section{Diseño del estudio}

Se realizó un estudio observacional, trasversal y analítico.

\section{Población y muestra}

La población de estudio estuvo conformada por los pacientes que acudieron al servicio de Oftalmología del Hospital II Vitarte (nivel 2) de EsSalud y esta fue de 2600 en el año 2014 e igualmente se estimó para el 2018.

Se utilizó la fórmula de cálculo de muestra de población finita. Para ambos años se consideró un adicional de 5\%. La selección de la muestra se realizó por muestreo aleatorio simple. 


\section{Criterios de selección}

Se incluyeron a aquellos pacientes asegurados nuevos o continuadores que aceptaron participar en el estudio mediante su conformidad en el consentimiento informado, que no presentaron alteraciones de la conciencia y capaces de comunicarse con claridad. Se excluyeron a aquellos pacientes en las que las encuestas no estuvieran resueltas correctamente 0 no hayan respondido a todas las preguntas.

\section{Variables e instrumentos de medición}

La técnica utilizada fue la encuesta y el instrumento usado fue un cuestionario que fue dividido el tres secciones: la primera, que incluía datos generales sociodemográficos, la condición del paciente y el tipo de seguro médico que el usuario poseía; la segunda, que correspondió al cuestionario de SERVQUAL, el cual está dividido en dos componentes (expectativas del entrevistado, percepciones del entrevistado) de 22 preguntas cada uno, en una escala de Likert de 1 al 7.

La tercera sección, solo realizada en el grupo del año 2018, incluía al cuestionario SERVQHOS que medía la calidad objetiva, subjetiva y satisfacción global del paciente a través de 19 preguntas cuantitativas y 7 cualitativas, en una escala de Likert que va de 1 (mucho más bajo de los estimado) a 5 (mucho más alto de los estimado).

\section{Análisis de los resultados}

La puntuación de satisfacción global se calculó según la media aritmética de cada uno de los bloques de preguntas y a su vez, con la media de los dos bloques de manera general, aplicándose estadísticas descriptivas; se utilizó la prueba de chi-cuadrado de Pearson $\left(X^{2}\right)$ en las comparaciones del grado de satisfacción (proporción de pacientes satisfechas/ insatisfechas) entre los diferentes años. El índice de satisfacción se obtuvo mediante la diferencia de la percepción general con la expectativa general.

El nivel de satisfacción del paciente con la calidad ${ }^{[13]}$ se midió mediante el índice de satisfacción, según la siguiente categorización: "nivel satisfecho" cuando el valor fue igual a 0 , "nivel muy satisfecho" cuando el valor fue $>0$ y "nivel insatisfecho" cuando el valor fue $<0$.

La puntuación de calidad global se calculó según la media aritmética de cada uno de los bloques de preguntas, y a su vez, con la media de los dos bloques de manera general. Así, puntuaciones inferiores a tres denotaron insatisfacción, puntuaciones iguales a tres señalaban conformidad con el servicio y expectativas cubiertas, pero no superadas; y aquellas puntuaciones mayores que tres, satisfacción. La valoración de la satisfacción global con la asistencia recibida se midió a través de las últimas preguntas (recomendación de acudir al centro de atención, el tiempo de ingreso, y las atenciones respetando las normas administrativas y la bioética de los profesionales sanitarios) con las mismas opciones de respuesta y otras direccionadas al trato humanizado. Se realizó estadística descriptiva y se utilizó la prueba de Chicuadrado $\left(X^{2}\right)$ de Pearson.
Se asumió un nivel de confianza del 95\% y se utilizó el paquete estadístico IBM SPSS Statistic Product and Service Solutions versión 25.0 .

\section{Aspectos éticos}

El estudio fue aprobado por el Comité de ética de la Universidad Nacional Mayor de San Marcos (UNMSM) (acta N 1861) y por el Comité de investigación institucional de EsSalud (carta N 2385 GRPA-ESSALUD-2019). La entrevista se realizó luego de que los usuarios aceptaron participar en el estudio, mediante la firma del consentimiento informado. Toda información recolectada se mantuvo cumpliendo la confidencialidad de los datos (todos fueron codificados).

\section{RESULTADOS}

Del total de 348 pacientes elegibles para el año 2014 y 363 para el 2018, se excluyó a 33 pacientes por no completar los cuestionarios. Así, se quedó con un total de 678 pacientes, los mismos que correspondían a 346 para el año 2014 (aplicación del cuestionario SERVQUAL) y 332 para el 2018 (aplicación de cuestionarios SERQUAL y SERVQHOS). La totalidad de las encuestas fueron debidamente llenadas, el cuestionario de SERVQUAL tuvo un alfa de Cronbach de 0,930 y el de SERVQHOS un Alfa de Cronbach =0,95; considerados como confiables.

Las características de los resultados sociodemográficos de ambas poblaciones se muestran en la Tabla 1, las comparaciones de satisfacción en las dimensiones de la calidad en la Tabla 2.

Tabla 1. Características sociodemográficas de todos los participantes.

\begin{tabular}{|c|c|c|c|}
\hline Características & $\begin{array}{l}\text { Total } \\
\text { n (\%) }\end{array}$ & $\begin{array}{c}2014 \\
(n=346)\end{array}$ & $\begin{array}{c}2018 \\
(n=332)\end{array}$ \\
\hline \multicolumn{4}{|l|}{ Sexo } \\
\hline Masculino & $305(45,0)$ & 160 & 145 \\
\hline Femenino & $373(55,0)$ & 186 & 187 \\
\hline Edad $(\bar{x} \pm D E)$ & $58,8 \pm 17,1$ & & \\
\hline \multicolumn{4}{|l|}{ Edad (años) } \\
\hline$<20$ & $10(1,5)$ & 5 & 5 \\
\hline $20-29$ & $53(7,9)$ & 25 & 28 \\
\hline $30-39$ & $99(14,7)$ & 49 & 50 \\
\hline $40-49$ & $145(21,6)$ & 66 & 79 \\
\hline $50-59$ & $120(17,8)$ & 64 & 56 \\
\hline $60-69$ & $108(16,1)$ & 51 & 57 \\
\hline$\geq 70$ & $137(20,4)$ & 80 & 57 \\
\hline \multicolumn{4}{|l|}{ Nivel de estudio } \\
\hline Analfabeto & $3(0,5)$ & 2 & 1 \\
\hline Primaria & $107(15,8)$ & 50 & 57 \\
\hline Secundaria & $269(39,7)$ & 153 & 116 \\
\hline Superior técnico & $180(26,6)$ & 83 & 97 \\
\hline Superior universitario & $118(17,4)$ & 57 & 61 \\
\hline \multicolumn{4}{|l|}{ Tipo de usuario } \\
\hline Nuevo & $128(19,6)$ & 53 & 75 \\
\hline Continuador & $525(80,4)$ & 268 & 257 \\
\hline
\end{tabular}

$\overline{\mathrm{X}}$ : media aritmética; DE: desviación estándar 
Tabla 2. Comparación de satisfacción global según las dimensiones de SERVQUAL 2014 y 2018, aplicando el X² con un IC al 95\%.

\begin{tabular}{|c|c|c|c|c|c|}
\hline \multirow[t]{2}{*}{ Dimensión } & \multicolumn{2}{|c|}{$\begin{array}{c}\text { Año } 2014 \\
(n=346)\end{array}$} & \multicolumn{2}{|c|}{$\begin{array}{c}\text { Año } 2018 \\
\text { (n=332) }\end{array}$} & \multirow[t]{2}{*}{ Valor de $\mathrm{p}^{*}$} \\
\hline & $n(\%)$ & Nivel & $n(\%)$ & Nivel & \\
\hline Fiabilidad & $141(40,7)$ & $\begin{array}{l}\text { Ligeramente } \\
\text { insatisfecho }\end{array}$ & $105(31,6)$ & $\begin{array}{l}\text { Ligeramente } \\
\text { insatisfecho }\end{array}$ & $p=0,014$ \\
\hline Capacidad de respuesta & $222(65,1)$ & Satisfecho & $82(24,7)$ & $\begin{array}{l}\text { Moderadamente } \\
\text { insatisfecho }\end{array}$ & $p<0,05$ \\
\hline Seguridad & $248(71,6)$ & Satisfecho & $123(37,0)$ & $\begin{array}{l}\text { Ligeramente } \\
\text { insatisfecho }\end{array}$ & $p<0,05$ \\
\hline Empatía & $218(63,0)$ & Satisfecho & $123(37,0)$ & $\begin{array}{l}\text { Ligeramente } \\
\text { insatisfecho }\end{array}$ & $p<0,05$ \\
\hline Aspectos tangibles & $209(60,4)$ & Satisfecho & $115(34,6)$ & $\begin{array}{l}\text { Ligeramente } \\
\text { insatisfecho }\end{array}$ & $p<0,05$ \\
\hline \multicolumn{6}{|c|}{ Satisfacción global (índice de calidad) } \\
\hline Percepciones (P) & \multicolumn{2}{|c|}{$5,44 \pm 0,35$} & \multicolumn{2}{|c|}{$5,20 \pm 0,35$} & - \\
\hline Expectativas (E) & \multicolumn{2}{|c|}{$5,37 \pm 0,21$} & \multicolumn{2}{|c|}{$5,98 \pm 0,08$} & - \\
\hline$\Delta(\mathrm{P}-\mathrm{E})$ & \multicolumn{2}{|c|}{0,07} & \multicolumn{2}{|c|}{$-0,78$} & - \\
\hline Nivel de satisfacción & \multicolumn{2}{|c|}{ Satisfecho } & \multicolumn{2}{|c|}{ Ligeramente insatisfecho } & - \\
\hline
\end{tabular}

* Según prueba de $\mathrm{X}^{2}$.

$\Delta(\mathrm{P}-\mathrm{E})$ : diferencia entre percepciones y expectativas.

Tabla 3. Calidad percibida en el 2018 por los pacientes $(n=332)$ instrumento SERVQHOS.

\begin{tabular}{|c|c|c|c|c|c|c|}
\hline \multirow[b]{2}{*}{ Variable } & \multicolumn{5}{|c|}{ Escala de Likert } & \multirow[b]{2}{*}{$x \pm D E$} \\
\hline & $\begin{array}{c}\text { Mucho peor } \\
(\%)\end{array}$ & $\begin{array}{l}\text { Peor } \\
(\%)\end{array}$ & $\begin{array}{c}\text { Como lo } \\
\text { esperaba (\%) }\end{array}$ & $\begin{array}{l}\text { Mejor } \\
(\%)\end{array}$ & $\begin{array}{l}\text { Mucho } \\
\text { mejor (\%) }\end{array}$ & \\
\hline \multicolumn{7}{|l|}{ Calidad objetiva } \\
\hline Tecnología biomédica & 0,9 & 5,1 & 41,6 & 37,3 & 15,1 & $3,61 \pm 0,84$ \\
\hline Aspecto del personal & 2,4 & 3,6 & 41,6 & 37,3 & 15,1 & $3,59 \pm 0,87$ \\
\hline Señalización intrahospitalaria & 1,5 & 4,5 & 39,5 & 39,5 & 15,0 & $3,62 \pm 0,85^{*}$ \\
\hline Puntualidad de las consultas & 1,8 & 5,4 & 36,1 & 37,3 & 19,4 & $3,67 \pm 0,91^{*}$ \\
\hline Comodidad de las habitaciones & 2,1 & 6,9 & 40,7 & 34,9 & 15,4 & $3,55 \pm 0,91$ \\
\hline Información dada por el médico & 0,6 & 2,4 & 39,2 & 41,3 & 16,5 & $3,71 \pm 0,79 *$ \\
\hline Tiempo de espera & 1,2 & 3,6 & 35,5 & 37,7 & 22,0 & $3,76 \pm 0,88^{*}$ \\
\hline Señalización extrahospitalaria & 3,0 & 4,8 & 38,3 & 36,7 & 17,2 & $3,60 \pm 0,93$ \\
\hline Información a familiares & 1,8 & 4,8 & 32,2 & 38,9 & 22,3 & $3,75 \pm 0,92 *$ \\
\hline \multicolumn{7}{|l|}{ Calidad subjetiva } \\
\hline Interés por solucionar problemas & 1,8 & 5,7 & 40,1 & 36,7 & 15,7 & $3,59 \pm 0,88$ \\
\hline Interés por cumplir promesas & 0,6 & 5,1 & 38,9 & 40,7 & 14,7 & $3,64 \pm 0,82 *$ \\
\hline Rapidez de respuesta & 1,5 & 7,8 & 36,7 & 41,0 & 13,0 & $3,56 \pm 0,87$ \\
\hline Disposición para la ayuda & 1,5 & 5,1 & 41,0 & 36,4 & 16,0 & $3,60 \pm 0,87$ \\
\hline Confianza y seguridad & 1,2 & 6,0 & 40,1 & 37,7 & 15,0 & $3,59 \pm 0,86$ \\
\hline Amabilidad del personal* & 1,2 & 5,4 & $43,7^{*}$ & 28,0 & 21,7 & $3,64 \pm 0,92^{*}$ \\
\hline Preparación del personal & 2,7 & 6,0 & 38,6 & 38,0 & 14,7 & $3,56 \pm 0,91$ \\
\hline Trato personalizado & 3,0 & 3,6 & 37,7 & 39,5 & 16,2 & $3,62 \pm 0,91$ \\
\hline Comprensión de necesidades & 0,9 & 5,4 & 34,3 & 41,0 & 18,4 & $3,70 \pm 0,86^{*}$ \\
\hline Interés de personal médico & 0,6 & 4,8 & 42,5 & 34,0 & 18,1 & $3,64 \pm 0,85^{*}$ \\
\hline
\end{tabular}

* Representa las más altas puntuaciones de satisfacción de la calidad objetiva, subjetiva y trato humanizado percibida por los pacientes atendidos en el año 2018 $\overline{\mathrm{X}}$ : media aritmética; DE: desviación estándar. 
Tabla 4. Calidad global medida por la satisfacción global según SERVQHOS.

\begin{tabular}{|c|c|c|c|c|}
\hline \multirow{2}{*}{ Variable } & \multicolumn{4}{|c|}{ Categoría (en \%) } \\
\hline & Muy satisfecho & Satisfecho & Poco satisfecho & Nada satisfecho \\
\hline \multirow[t]{2}{*}{ Satisfacción global } & 7,5 & 63,6 & 28,0 & 0,9 \\
\hline & & Sin dudarlo & Tengo dudas & Nunca \\
\hline \multirow{2}{*}{\multicolumn{2}{|c|}{ ¿Recomendaría este hospital a otras personas? }} & 50,6 & 49,1 & 0,3 \\
\hline & & $\begin{array}{c}\text { Menos de lo } \\
\text { necesario }\end{array}$ & $\begin{array}{l}\text { El tiempo } \\
\text { necesario }\end{array}$ & $\begin{array}{l}\text { Más de lo } \\
\text { necesario }\end{array}$ \\
\hline \multirow{2}{*}{\multicolumn{2}{|c|}{ A su juicio, ¿̇ha ingresado en el hospital? }} & 16,0 & 81,0 & 3,0 \\
\hline & & & Sí & No \\
\hline \multicolumn{3}{|c|}{ ¿Le han realizado en el hospital pruebas o intervenciones sin pedir permiso? } & 2,7 & 97,3 \\
\hline \multicolumn{3}{|c|}{ ¿Conoce el nombre del médico que lo atendía habitualmente? } & 82,5 & 17,5 \\
\hline \multicolumn{3}{|c|}{ ¿Conoce el nombre de la enfermera que lo atendía habitualmente? } & 24,1 & 75,9 \\
\hline \multicolumn{3}{|c|}{ ¿Cree que ha recibido suficiente información sobre lo que pasaba? } & 70,2 & 29,8 \\
\hline
\end{tabular}

Se realizó las pruebas de $\mathrm{X}^{2}$ de Pearson para las comparaciones de los resultados. No hubo diferencias significativas en cuanto al sexo $(p=0,48)$, respecto al grupo etario, el más frecuente en el 2014 fue el mayor de 70 años y el de 40-49 años en el 2018. No hubo diferencia en la proporción de niveles de estudio $(p=0,1)$; ni el tipo de usuario $(p=0,05)$. No hubo diferencias en cuanto a las características de la población (sexo, nivel de estudio, tipo de usuario) entre ambos años. Esto indicaría que ambas muestras son similares y comparables.

Los resultados muestran el contraste entre el año 2014 y el 2018, la satisfacción global en el año 2014 frente a la ligera insatisfacción global del año 2018, con una diferencia estadísticamente significativa en todas las dimensiones, destacándose la moderada insatisfacción en la capacidad de respuesta.

Respecto a las percepciones, los pacientes estuvieron satisfechos con la calidad objetiva y de estas destacan el tiempo de espera, la información que les da el médico tanto individual como a sus familiares, y la señalización de los ambientes intrahospitalarios. Respecto a la calidad subjetiva destaca la comprensión de sus necesidades, el interés por cumplir promesas, la amabilidad y el interés del personal médico (Tabla 3).

Para determinar la calidad global percibida por el usuario externo en el año 2018, se utilizaron las preguntas finales de SERVQHOS sobre la recomendación de este centro de atención, el tiempo de ingreso, y las atenciones respetando las normas administrativas y la bioética de los profesionales sanitarios, los resultados se observan en la Tabla 4.

La satisfacción global mostrada está relacionada con el respeto del personal de salud, que le muestran al paciente al solicitarle el permiso para las intervenciones médicas y la comunicación personalizada con su médico, recomendando al hospital sin dudarlo en más de la mitad de los pacientes.
Se realizó la prueba de Pearson para estimar la correlación entre el puntaje de satisfacción global del instrumento de SERVQUAL y el puntaje de calidad global de SERVQHOS año 2018, y se obtuvo un coeficiente de correlación $r=0,132(p=0,01)$ lo que se muestra en la Figura 1. No es una correlación perfecta, pero si tiene puntos de coincidencia que permiten establecer una correlación positiva débil en la medición de la calidad por la satisfacción del usurario.

\section{DISCUSIÓN}

En nuestro estudio utilizamos dos instrumentos en el año 2018 para medir la satisfacción del paciente como indicador directo de la calidad del servicio recibido; sin embargo, debemos considerar que los factores que definen la calidad son complejos. El modelo multidimensional usado por los cuestionarios de SERVQUAL ${ }^{[2,3,6,12]}$ y SERVQHOS ${ }^{[13,14]}$ para el análisis de las brechas del servicio, abarca elementos básicos de la evaluación de la calidad global de la atención sanitaria, agrupándolos en aspectos relacionados con la calidad objetiva (procesos y elementos tangibles) y aspectos relacionados a la calidad subjetiva (profesionalismo, ética y trato humanizado).

Teóricamente, ambos instrumentos miden calidad y si son utilizados por un mismo usuario, el resultado debería ser igual o semejante; no obstante, se debe considerar que algunos aspectos podrían influenciar a priori en lo esperado, tales como el momento de la toma de datos, las experiencias previas del paciente, el estilo de vida, la cobertura sanitaria y su educación.

En cuanto a la satisfacción global del año 2014, los resultados son similares a estudios publicados. Calderón ${ }^{[15]}$ concluyó que el nivel de satisfacción se relacionaba con la calidad de atención del servicio de laboratorio clínico en sus diferentes dimensiones. 


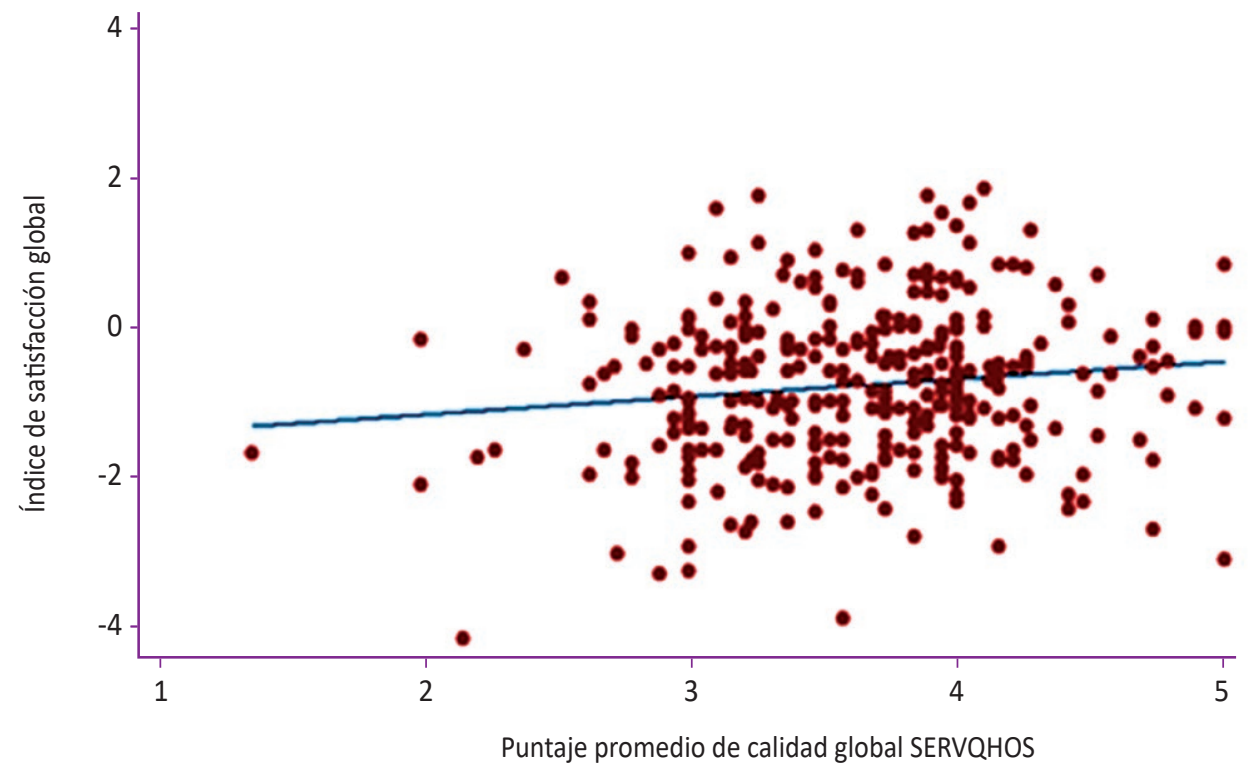

Figura 1. Correlación entre la calidad global de SERVQHOS y la satisfacción global de SERVQUAL $(r=0,132)$ en la población de 332 pacientes en el año 2018.

Piedra ${ }^{[16]}$ realizó un estudio en el tópico de medicina de un hospital local y encontró que el $85,5 \%$ estaba satisfecho con el servicio, siendo la dimensión empatía (91,9\%) y fiabilidad $(90,3 \%$ ) los más valorados. Ramírez ${ }^{[17]}$ realizó un estudio similar en un hospital de nivel II-2 del interior del país y encontró una satisfacción global positiva del paciente y las dimensiones destacables fueron fiabilidad y seguridad, demostrando la relación positiva entre la satisfacción del paciente y la calidad del servicio recibido.

Nuestros resultados del año 2018, aplicando el modelo SERVQUAL, son similares a lo reportado por Delgado ${ }^{[18]}$. Se encontró una insatisfacción global leve, con énfasis en las dimensiones de capacidad de respuesta y elementos tangibles; sin embargo, es destacable la empatía del profesional de salud, que los distingue en el proceso de atención.

Mamani ${ }^{[19]}$, en su estudio con una muestra similar en el Hospital II -1 MINSA, reportó insatisfacción en el paciente de consultorio externo; de las cinco dimensiones, la capacidad de respuesta $(76,10 \%)$ y aspectos tangibles $(82,26 \%)$ fueron los más relevantes y recomendó implementar el plan de mejora de calidad. Obtuvimos resultados similares en el año 2018.

Aragón ${ }^{[20]}$ encontró resultados con un nivel bajo de satisfacción, lo que coincide con nuestro estudio en el 2018. Igualmente, Robles ${ }^{[7]}$ identificó una insatisfacción global leve. con dimensiones que deben mejorarse como la capacidad de respuesta, coincidencias para la implementación de evaluaciones periódicas de la calidad por parte de los directivos de gestión de la calidad para establecer y supervisar procesos de mejora continua, principalmente en la obtención de citas y la información del profesional de salud.

Huanchaco ${ }^{[21]}$ encontró resultados de insatisfacción global moderada en los pacientes de los servicios de cirugía y medicina, las dimensión de mayor insatisfacción fue la capacidad de respuesta, que está relacionada con la atención en el área de acreditación para su hospitalización, farmacia, laboratorio y toma de imágenes (quejas relacionadas a la distancia del hogar o centro de labor), sistema de traslado usuarios que requieren sillas de rueda, la falta de insumos radiológicos, reactivos químicos y la falta de recurso humano asistencial, logístico y administrativo.

Durante el periodo 2014 - 2018 la población asegurada adscrita a la institución aumentó, pero la infraestructura es la misma. Cada año la fiscalización de centros asistenciales, con la finalidad de proteger los derechos en salud de cada peruano, se hace más eficiente por la supervisión de la Superintendencia de Salud - SUSALUD, que empodera al ciudadano para manifestar su disconformidad con los servicios recibidos lo que se ve reflejado en los resultados y el análisis de la investigación en el 2018.

Al utilizar un instrumento (SERVQHOS) que mide la calidad global mediante preguntas con respuestas no numéricas sino cualitativas que indagan la expectativa del servicio, los pacientes aparentemente fueron más permisivos y establecieron una mejor comunicación desde su percepción de la gestión administrativa, profesionalismo médico y las consideraciones éticas de trato humanizado por parte del personal asistencial.

El cuestionario SERVQHOS orienta mucho mejor a los usuarios y determina la decisión de recomendar el servicio ofertado. En este sentido nuestra investigación tuvo los resultados de satisfacción global en el 71\%; sin embargo, solo el 50\% recomendaría el servicio. La duda de la proporción restante se podría atribuir a la gran demanda y la poca oferta de servicios asistenciales. Estos resultados son similares a lo que obtuvo Braña-Marcos et al. ${ }^{[22]}$, quien desarrolló un estudio multicéntrico sobre la satisfacción de mujeres con diagnóstico de cáncer y la atención 
recibida; su satisfacción estuvo asociada a la calidad objetiva de la comunicación clara y precisa del médico y la insatisfacción relevante fue la comunicación deficiente con sus familiares. En cuanto a la calidad subjetiva, estuvieron satisfechas con la amabilidad del personal asistencial y la insatisfacción fue con el tiempo de espera para la consulta.

Similares resultados obtuvo Ariza-Hernández ${ }^{[23]}$, quien utilizando el instrumento de SERVQHOS en un hospital público encontró que la mayoría estuvo satisfecha con la atención médica y recomendaría el hospital; el acto médico fue lo más valorado, la comunicación asertiva y empática del profesional, y la gestión administrativa para facilitar los trámites de obtención de citas; sin embargo, la insatisfacción fue notoria en los elementos tangibles, limpieza y disponibilidad de los elementos de higiene, papeles y jabón para lavarse las manos, las incomodidades y mal estado de las sillas de espera. Estas percepciones al ser dispuestas en la gerencia permiten la mejora continua de la calidad y evitar el deterioro de la imagen pública del hospital.

Pérez Cantó ${ }^{[24]}$, utilizando el SERVQHOS, hizo un estudio comparativo en dos centros asistenciales, uno público y otro privado, para evaluar la gestión sanitaria. Sus resultados mostraron que los índices de satisfacción eran elevados, con escasas diferencias entre ambos hospitales y superiores en ciertas áreas a otros estudios. Aparecieron como predictores de calidad factores como tiempo, agilidad de los sistemas e información, posiblemente por los cambios en las necesidades y expectativas de los usuarios. Fueron escasas las experiencias anteriores que compararan la satisfacción entre modelos de gestión diferentes como este estudio, lo que nos lleva a reflexionar en la medición de calidad global como mejor predictor para una gestión de calidad.

Arrébola-Pajares et al. ${ }^{[25]}$, en la investigación realizada sobre la satisfacción del paciente atendido en Urología de un hospital público en España, encontró que el 95,4\% calificó su nivel de satisfacción global como satisfactoria o muy satisfactoria. Los aspectos subjetivos mejor calificados fueron la amabilidad del personal y el trato personalizado. Destaca como aspecto objetivo peor valorado el estado de las habitaciones; las variables relacionadas con una mayor satisfacción global fue el menor tiempo de estancia hospitalaria, conocer el nombre de la enfermera, la información recibida y que recomendaría el hospital sin dudarlo.

Consideramos necesario seguir investigando en este campo y tener más estudios con el instrumento de SERVQHOS en los diferentes centros asistenciales y con especial interés en el servicio de oftalmología. Creemos que se debe continuar con la medición de satisfacción del usuario y la calidad percibida como un proceso continuo y dinámico que requiere el compromiso de todos los actores del servicio brindado para alcanzar la calidad y la seguridad en la atención de salud brindada.

Concluimos que en el año 2014 el nivel de satisfacción del paciente respecto a la calidad del servicio recibido en oftalmología fue "me encuentro satisfecho", mientras que en el 2018 fue "ligeramente insatisfecho"; y en la medición de la calidad global los pacientes estuvieron satisfechos, destacándose la atención personalizada del médico y el respeto mostrado a los pacientes. La satisfacción global de SERVQUAL con la calidad global de SERVQHOS presentaron una correlación positiva.

Contribuciones de autoría: ambas autoras participaron en la formulación del problema de investigación, desarrollo de esta, redacción de artículo y aprobación de la versión final del mismo.

Fuente de financiamiento. Autofinanciado por las autoras.

Potenciales conflictos de interés. Los autores declaran no tener conflictos de intereses.

Agradecimientos. A la gerencia del hospital por brindar el permiso para realizar la investigación y a las Unidad de Posgrado de la UNMSM por el respaldo administrativo.

\section{ORCID}

Nora Beatriz Mendoza Díaz, https://orcid.org/0000-0002-0438-2304 Maritza Placencia Medina, https://orcid.org/0000-0003-3624-3461

\section{REFERENCIAS BIBLIOGRÁFICAS}

1. Dirección General de Salud de las Personas. Documento técnico RM 519-2006/MINSA, Sistema de gestión de la calidad en Salud [Internet]. Lima: MINSA; 2007 [citado el 10 octubre de 2019]. Disponible en: http://bvs.minsa.gob.pe/local/dgsp/000_ SGCALIDAD-1.pdf

2. Parasuraman A, Zeithaml V, Berry L. A conceptual model of service quality and its implications for future research. J Mark. 1985;49(4):41-50. doi: 10.1177/002224298504900403

3. Cabello E, Chirinos JL. Validación y aplicabilidad de encuestas SERVQUAL modificadas para medir la satisfacción de usuarios externos en servicios de salud. Rev Med Hered. 2012;23(2):88-95.

4. Seguro Social del Perú (EsSalud). Programa de calidad de servicio [Internet]. Lima: EsSalud; 2008 [citado el 10 octubre de 2019]. Disponible en: http://www.essalud.gob.pe/defensoria/manual calidad.pdf

5. Donabedian, A. Evaluación de la calidad de la atención médica. Rev Calidad Asistencial. 2001;16:S11-S27.

6. Parasuraman A, ZeithamI VA, Berry LL. SERVQUAL: a multiple-item scale for measuring consumer perceptions of services quality. Journal of Retailing. 1988:64(1):12-40.

7. Robles I, Placencia M, Carreño R. Satisfacción del usuario externo de neurocirugía del Instituto Nacional de Ciencias Neurológicas, Lima - Perú, 2016. Horiz Med. 2019;19(3):58-71. doi: 10.24265/ horizmed.2019.v19n3.09

8. Torres $\mathrm{M}$, Vásquez $\mathrm{C}$. Modelos de evaluación de la calidad del servicio: caracterización y análisis. Compendium. 2015;18(35):57-76.

9. Cruz Arlet, Orduña M, Álvarez J. Evolución del concepto de calidad y los modelos de medición de calidad en el servicio. Innovaciones de Negocios. 2019;15(30):259-78

10. Castro H. Efectividad del Sistema de Gestión de la calidad nivel de los servicios de salud en el Hospital I ESSALUD - Tingo Maria 2016 [tesis de doctorado]. Huánuco, Perú: Universidad de Huánuco; 2019. 
11. Tobón L, Cardona J. Evaluación de la calidad de los servicios médicos según las dimensiones del SERVQUAL en un hospital de Colombia. Archivos de medicina. 2018;14(4):9 p. doi: 10.3823/1402

12. Ibarra L, Espinoza B. Servqual, una propuesta metodológica para evaluar la percepción de la calidad. RelbCi. 2014; 1(4):107-20.

13. Mira JJ, Aranáz J, Rodríguez M, Buil AA. SERVQHOS: un cuestionario para evaluar la calidad percibida de la asistencia hospitalaria. Med Prev [Internet]. 1998 [citado 20 enero 2020];IV:12-18. Disponible en: http://calite-revista.umh.es/indep/web/art_servqhos.pdf

14. Barragán J, Manrique F. Validez y confiabilidad del Servqhos para enfermería en Boyacá, Colombia. Av Enferm. 2010;28(2):48-61.

15. Calderón R. Satisfacción del paciente y la calidad de atención del Servicio de Laboratorio Clínico en el Hospital de la Solidaridad Tacna, enero del 2018 [tesis de maestría]. Lima, Perú: Universidad Privada Norbert Wiener; 2018.

16. Piedra I. Calidad de atención y el grado de satisfacción de los pacientes atendidos en el Tópico de Medicina del Servicio de Emergencia del Hospital II-EsSalud Vitarte 2017 [tesis de maestría]. Lima. Perú: Universidad Nacional Mayor de San Marcos; 2019.

17. Ramírez R. Calidad de atención de salud y su relación con la satisfacción del usuario de la consulta externa en el Hospital Hugo Pesce Pescetto de Andahuaylas, 2014 [tesis de pregrado]. Andahuaylas, Perú: Universidad Nacional José María Arguedas; 2015.

18. Delgado P. Modelo SERVQUAL en la determinación de la calidad del servicio de los pacientes de consulta externa hospital universitario, ciudad de Guayaquil [tesis de pregrado]. Guayaquil, Ecuador: Universidad laica Vicente Rocafuerte; 2019.
19. Mamani L. Nivel de satisfacción en la atención de consulta externa, en pacientes del Hospital II - 1 - MINSA - Ilo, 2017 [tesis de pregrado]. Moquegua, Perú: Universidad José Carlos Mariátegui; 2017.

20. Aragón A. Calidad de cuidado enfermero y nivel de satisfacción del paciente del servicio de Medicina del Hospital Carlos Monge Medrano, Juliaca - 2015 [tesis de pregrado]. Juliaca, Perú: Universidad Peruana Unión; 2015.

21. Huanchaco M. Nivel de satisfacción de los usuarios hospitalizados en los servicios de medicina y cirugía del Hospital Nacional. Guillermo Almenara Irigoyen, EsSalud, Lima-Perú, 2016 [tesis de maestría]. Lima, Perú: Universidad César Vallejo; 2017.

22. Braña-Marcos B, Carrera-Martínez D, De la Villa-Santoveña M, Avanzas-Fernández S, Gracia-Corbato M, Vegas-Pardavila E. Análisis de satisfacción en mujeres con reciente diagnóstico de cáncer de mama. Estudio multicéntrico en Servicios de Oncología. Rev Calid Asist. 2011;26(5):306-14. doi:10.1016/j.cali.2011.04.006

23. Godínez F, Reyes R, Ariza F, Maruris M, Cuevas A, Cabañas E. Nivel de satisfacción de usuarios de consulta externa en un hospital público. Psicología y Salud. 2014;24(1):97-107. doi: 10.25009/pys. v24i1.724

24. Pérez V, Maciá ML, González VM. Satisfacción de los usuarios en 2 hospitales con diferente modelo de gestión. J Healthc Qual Res. 2018;33(6):334-42. doi:10.1016/j.jhqr.2018.07.005

25. Arrebola-Pajares A, Tejido-Sanchez A, Jiménez-Alcaide E, MedinaPolo J, Perez-Cadavid S, Guerrero-Ramos F, et al. Survey of satisfaction in hospitalized patients at a urology department. Arch Esp Urol. 2014;67(7):621-7. 